\title{
Predicting Arachnoid Membrane Descent in the Chias- matic Cistern in the Treatment of Pituitary Macroadenoma
}

\author{
Hak Cheol Ko, Seung Hwan Lee, Hee Sup Shin, Jun Seok Koh \\ Stroke and Neurological Disorders Centre, Kyung Hee University Hospital at Gangdong, College of Medicine, Kyung Hee University, Seoul, \\ Korea
}

Objective : Preoperative prediction of the arachnoid membrane descent in pituitary surgery is useful for achieving gross total removal and avoiding cerebrospinal fluid leakage resulting from tearing of the arachnoid membrane in the chiasmatic cistern. In this study, we analyzed the patterns of arachnoid membrane descent during or after pituitary tumor surgery and identified the factors related to this descent.

Methods : Analysis was restricted to pituitary macroadenomas not extending into the third ventricle or over the internal carotid artery. To minimize confounding factors, patients who underwent revision surgery, those who had a torn arachnoid during operation or small medial diaphragma sellae (DS) opening, and subtotal resections were excluded. We enrolled 41 consecutive patients in this retrospective analysis. The degree of arachnoid descent was categorized using intraoperative videos. Preoperative magnetic resonance findings, including tumor height, suprasellar extension, and variables including DS area and medial opening size, tumor composition, and displacement of the pituitary stalk and gland were evaluated to determine their correlations with arachnoid membrane descent.

Results : Arachnoid membrane descent was significantly correlated with DS area and medial opening size. Based on T2-weighted images (T2WI) magnetic resonance (MR) images, tumor composition was significantly associated with arachnoid membrane descent. Other factors were not significantly correlated with arachnoid membrane descent.

Conclusion : T2WI of tumor composition and preoperative MR imaging of DS area and medial opening provided valuable information regarding arachnoid membrane descent. These parameters may serve as fundamental measures to facilitate complete resection of pituitary macroadenomas.

Key Words : Pitutiary neoplasm · Pituitary adenoma · Diaphragma sellae · Arachnoid mater · Medial opening.

\section{INTRODUCTION}

The use of endoscopy in pituitary tumor surgery has facilitated ease of access to the sellar and parasellar regions by pro- viding a wider and more precise view. Accordingly, the demand for improving the precision of pituitary surgery and broadening accessible areas using the transspenoidal approach (TSA) has grown. Recent literature has provided a detailed

- Received : May 15, 2020 •Revised : June 1, 2020 •Accepted : June 2, 2020

- Address for reprints : Seung Hwan Lee

Stroke and Neurological Disorders Centre, Kyung Hee University Hospital at Gangdong, College of Medicine, Kyung Hee University, 892 Dongnam-ro, Gangdong-gu, Seoul 05278, Korea

Tel : +82-2-440-6286, Fax : +82-504-005-1530, E-mail : toast2000@hanmail.net, ORCID : https://orcid.org/0000-0001-8043-632X

This is an Open Access article distributed under the terms of the Creative Commons Attribution Non-Commercial License (http://creativecommons.org/licenses/by-nc/4.0) which permits unrestricted non-commercial use, distribution, and reproduction in any medium, provided the original work is properly cited. 
anatomical dissection of the pituitary gland and cavernous sinus for achieving complete removal of the tumor with avoidance of complications ${ }^{2,3,7-9,11,14,15)}$. However, the issue of cerebrospinal fluid (CSF) leakage, which must be predicted preoperatively and adequately sealed, remains. Despite advances in sealing CSF leakage, complete prevention of this phenomenon would be optimal. In most pituitary macroadenomas, which reside in the sellar turcica, CSF leakage may be preventable if precise information on normal and abnormal structures is acquired beforehand, though giant pituitary macroadenomas requiring an extended TSA typically accompany CSF leakage.

A significant amount of tumors in the suprasellar portion may remain when dissection above the tumor is not sufficiently performed in efforts to avoid CSF leakage. The issue of whether to perform more extensive exploration and dissection arises when the suprasellar structure does not descend either with the tumor or arachnoid membrane. Further, verification and resection of the dorsal portion of the tumor is hindered when the suprasellar structure drops prematurely (prolapses). In this context, preoperative estimation of the descent of the suprasellar structure in pituitary macroadenomas would facilitate successful TSA with complete resection.

Suprasellar structures associated with pituitary adenomas are composed of multiple layers, which include the pseudocapsule of the tumor, normal pituitary gland and stalk, diaphragma sellae (DS), and arachnoid membrane of the chiasmatic cistern (Fig. 1). DS is a component of dura mater, which covers the sellar turcica and is a landmark layer indicating whether the tumor extends suprasellarly. An opening known as the medial opening exists in the center of DS, through which the pituitary stalk passes from the hypothalamus to the posterior pituitary gland. Pituitary adenoma growth widens this opening to become a passage to the suprasellar region. Conversely, the arachnoid membrane with or without the pseudocapsule often (but not always) descends through this opening when the prop of the tumor is broken during surgery.

In this study, we sought to elucidate the factors predicting arachnoid membrane descent in the chiasmatic cistern, which would facilitate the prevention of CSF leakage and complete tumor removal. For practical use in surgery, the study was analyzed based on findings, which were routinely used as preoperative diagnostic tools.

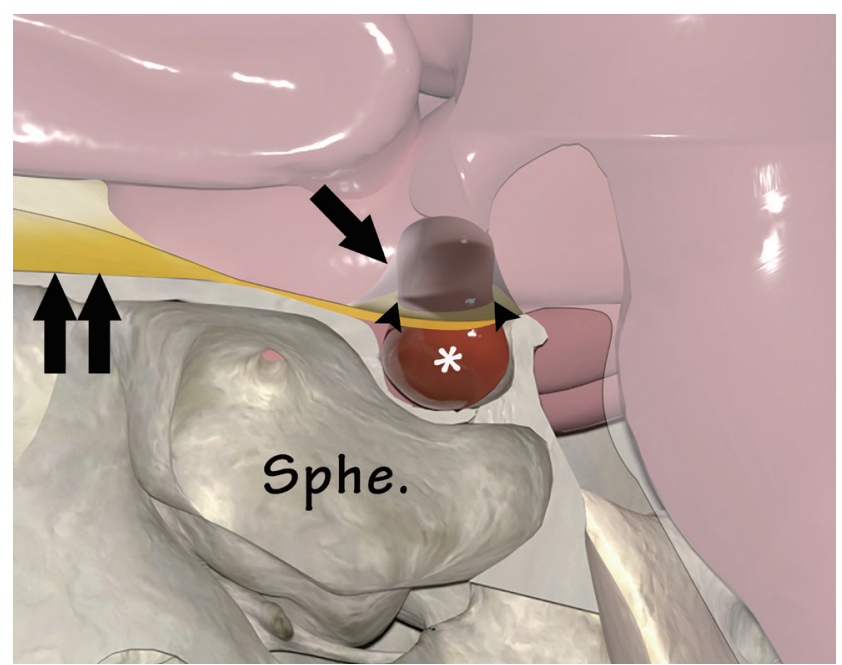

Fig. 1. Illustration depicting layers of suprasellar structures associated with pituitary adenoma (asterisk) (sagittal section). Pituitary adenoma grows toward the chiasmatic cistern through the medial opening of the diaphragma sellae (arrowheads), which is extention of dura mater (double arrow), forming the roof of the sellar turcica. Note that the arachnoid membrane (arrow) in the cistern is elevated and attached to the tumor surface due to the upward growth of the pituitary adenoma. Sphe. : sphenoid sinus.

\section{MATERIALS AND METHODS}

This study was approved by the Ethics Committee of Kyung Hee University Hostpital at Gangdong (IRB Approval No. KHNMC 2020-05-017). Since this was a retrospective medical record review involving de-identified patient data, patient consent was not required for this study. Of 74 patients who underwent endoscopic TSA surgery for pituitary adenoma resection between January 2016 and December 2018 at our institution, 41 patients were enrolled after excluding patients who required revisional TSA surgery (12 patients), patients with adenomas remaining in the sellar turcica without widening of the medial opening of DS (nine patients), those with expanding macroadenomas reaching the third ventricle and over the internal carotid artery (eight patients), and cases in which we were unable to assess the descent of the arachnoid membrane due to an arachnoid tear during surgery (four patients). Adenomas were diagnosed based on pathophysiological findings.

The study period was specifically chosen based on the period when the strategy for adenoma removal was changed from initial internal debulking followed by pseudocapsule removal to initial extra-capsular dissection followed by tumor debulking. This is because the former approach hindered verification 
of arachnoid membrane descent due to incomplete removal of the pseudocapsule or led to tearing of the arachnoid membrane during dissection. The medical records of the patients were retrospectively reviewed, including the patients' clinical status, imaging findings pre- and post-surgery, and intra-operative videos. Particular attention was paid to the analysis of intra-operative videos for precise assessment of the arachnoid membrane descent, as materials filling the tumor bed for hemostasis after surgery may have interfered with the evaluation of arachnoid membrane descent on postoperative magnetic resonance (MR) imaging.

Prior to surgery, patients underwent endocrinologic, ophthalmologic, and neurological evaluations, as well as MR imaging. MR images included T1-weighted (T1W) sagittal images with and without enhancement, T1W coronal images with enhancement, and T2W coronal images. T1W coronal dynamic sequences $(0,30,60,90$, and 120 seconds after contrast injection) were evaluated to delineate the exact location of the tumor and normal pituitary gland. Slice thicknesses were 2 $\mathrm{mm}$ and $2.5 \mathrm{~mm}$ on plain and dynamic sequences, respectively (3.0 Tesla system, Achieva; Philips Healthcare, Best, the Netherlands). Postoperative MR imaging was performed within 48 hours after surgery.

All tumor morphology parameters were assessed on preoperative MR imaging. The height of the tumor, suprasellar extension height, cavernous sinus involvement, properties of the internal tumor content, deviation of the normal pituitary stalk and gland, and DS morphology were analyzed. Infiltration of the cavernous sinus was defined as Knosp grade 3 or 4 . The height of suprasellar extension was measured on T2W coronal section images, which displayed the DS as a distinctive dark line, by drawing a line on the medial edge of the medial opening of the DS and measuring the maximal superior end of the tumor perpendicular to the line. Tumor composition was determined based on T2-weighted images (T2WI) and were grouped as follows : group A, diffusely mixed; group $\mathrm{B}$, mixed with some cystic component; and group C, homogenous.

Morphological analysis of DS was based on the assumption that the shape of the medial opening was oval, and the DS was square. The area of the medial opening was calculated using the formula for the area of an oval :

$$
\mathrm{A}=\pi \times \frac{\mathrm{W}}{2} \times \frac{\mathrm{V}}{2}
$$

where $\mathrm{A}=$ area, $\pi=3.14$, $\mathrm{W}=$ width, and $\mathrm{V}=$ length of the vertical line in the medial opening. The ' $V$ ' parameter was extracted from checkpoint64 (Stratovan Corporation, Davis, CA, USA) to construct a sagittal T2WI from coronal T2WI.

As such, the area of DS was calculated using the following formula for a square :

$$
\mathrm{A}=\mathrm{W} \times \mathrm{V}
$$

where $\mathrm{A}=$ area of $\mathrm{DS}, \mathrm{W}=$ width (between the most superior end of the medial wall of the cavernous sinus), and V=length of the vertical line (between the tuberculum sellae and dorsum sellae) in the DS.

A ratio of the areas of the medial opening and DS was calculated and entered as a variable for the analysis.

The locations of displaced pituitary stalks and glands were examined and classified as follows : type A, pituitary stalk elevated within the midline and a strongly enhanced thin structure (assumed to be a flattened gland) evenly distributed on both sides of the tumor; type B, the pituitary stalk and flattened gland deviated only on one side of the tumor (laterally or posteriorly); and type $\mathrm{C}$, the pituitary stalk deviated only on one side with a flattened gland on both sides.

In the analysis of intra-operative clips, we conducted a thorough inspection of each surgery, focusing on tumor consistency (soft, soft with mixed contents, fibrous with mixed contents, and fibrous) and the degree of arachnoid membrane descent during or after tumor removal. The findings of intraoperative arachnoid membrane descent after tumor removal were graded as follows : no descent, remained at its location without descent despite verification of oscillation of the roof structure; minimal descent, the roof structure descended minimally; moderate, the structure descended as much as the height of the suprasellar extension through the medial opening; and marked descent, the structure descended more than the extension height and the descended arachnoid membrane was so swollen that it appeared transparent.

Based on the degree of arachnoid membrane descent verified in the intra-operative clips, the parameters were compared and analyzed for correlations between arachnoid membrane descent and other parameters.

\section{Statistical analysis}

Statistical analysis was performed using commercial software (SPSS version 25.0; IBM Corp., Armonk, NY, USA). The significance level was set at $p<0.05$. As the degree of arachnoid 


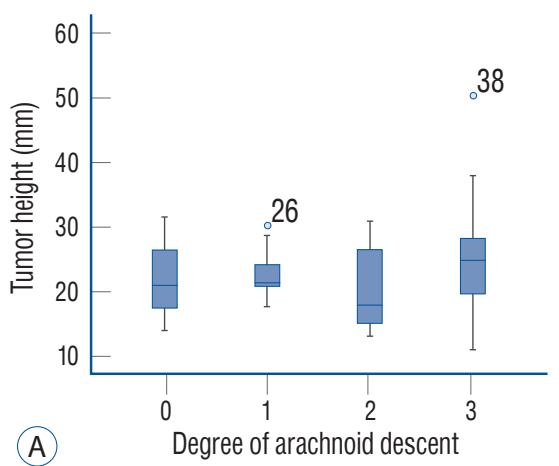

(A)
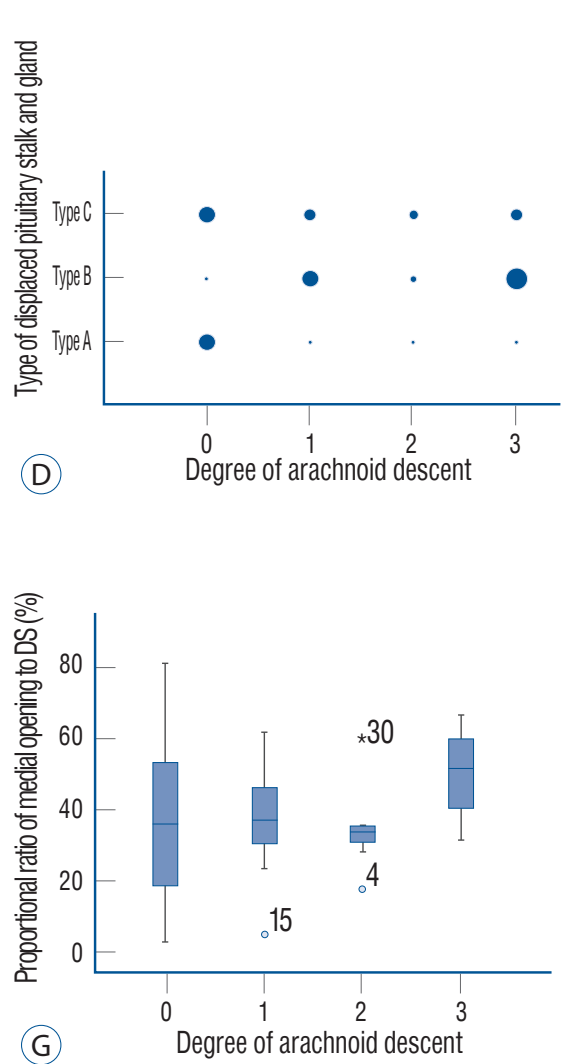
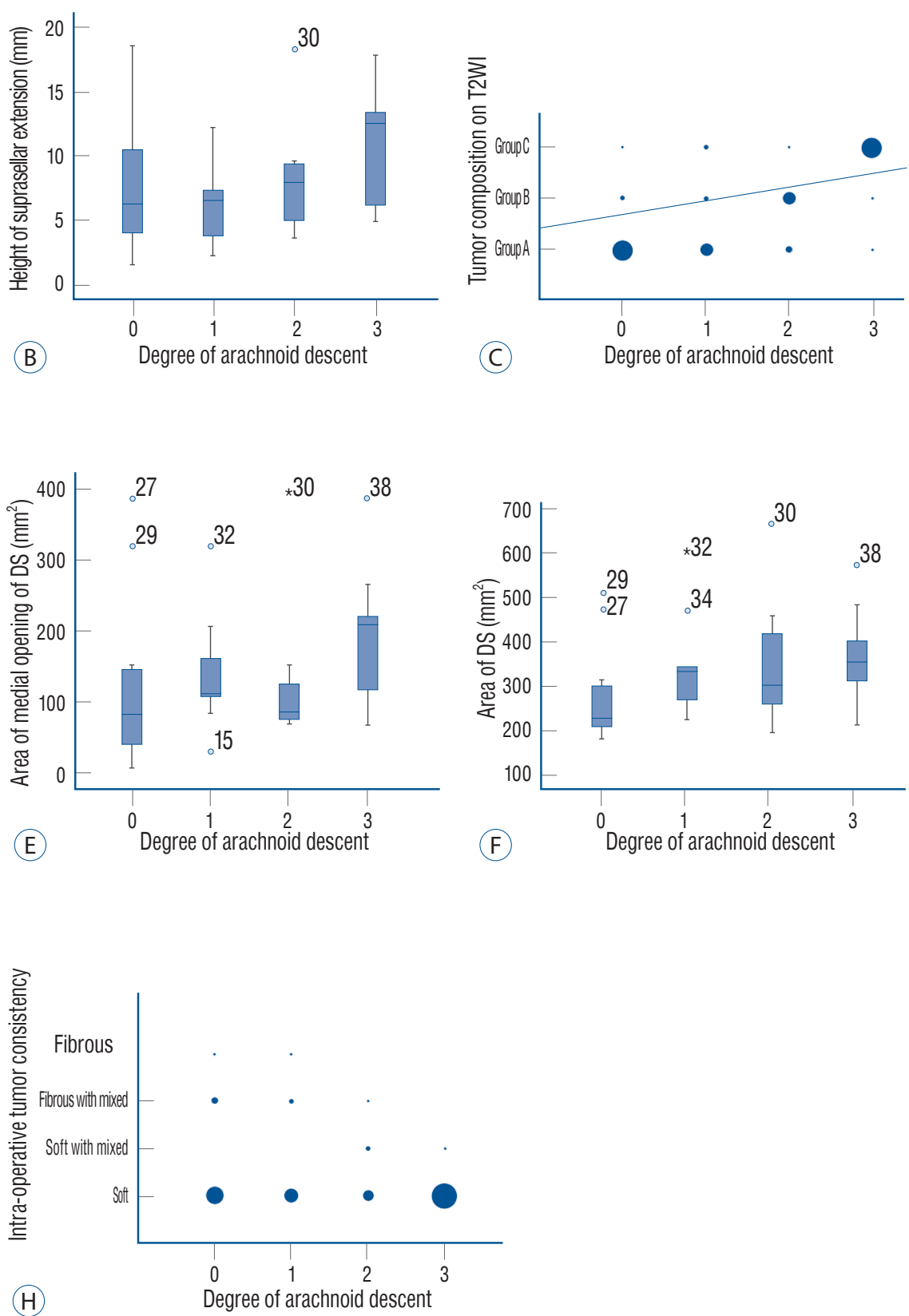

Fig. 2. Boxplots and volumetric scatter plots of the variables compared to the degree of arachnoid membrane descent after tumor removal. The categorical variables are depicted with volumetric scatter plots, and continuous variables are presented with boxplots. A : Boxplot of tumor height compared to the degree of arachnoid membrane descent; no correlation $(r=0.091, p=0.588)$. B : Boxplot of the height of the suprasellar extension on pre-operative MR imaging with the degree of arachnoid membrane descent showed a trend towards a correlation, but this was not statistically significant $(r=0.285, p=0.083)$. C : Volumetric scatter plot of tumor composition on pre-operative T2WI compared to the degree of arachnoid membrane descent revealed a strong positive correlation $(r=0.571, p<0.01)$. $D$ : The displacement of the pituitary gland and stalk on pre-operative MR imaging compared to arachnoid membrane descent is presented with the volumetric scatter plot, demonstrating no correlation $(r=0.0008, p=0.962)$. $E$ and $F$ : Boxplots of the area of the medial opening and DS compared to arachnoid membrane descent. Both areas were correlated with the degree of arachnoid membrane descent (area of the medial opening, $r=0.340, p=0.037$; area of $D S, r=0.348, p=0.032$ ), although the ratio of the two areas ( $G$ ) was not $(r=0.241$, $p=0.145) . \mathrm{H}$ : The intra-operative consistency of the tumor depicted with a volumetric scatter plot was not correlated with arachnoid membrane descent $(r=-0.299, p=0.073)$. T2WI : T2-weighted images, DS : diaphragma sellae, MR : magnetic resonance. 
membrane descent was a categorical variable, correlation analysis was performed using the Spearman rank-order correlation coefficient.

\section{RESULTS}

This study included 22 male and 19 female patients with a mean age of 58.7 years (range, 28 to 83 years). Most pituitary adenomas were non-functioning tumors (39 patients), and their presenting symptoms were visual field defects (14 patients), headache (13 patients), hypopituitarism (seven patients), oculomotor nerve palsy (four patients), and dizziness (one patient). Two patients had growth-hormone secreting adenomas, which manifested with acromegalic features. There were 13 patients $(31.7 \%)$ with no descent, nine patients $(22.0 \%)$

Table 1. Categorical variables according to degree of arachnoid descent and their correlations

\begin{tabular}{|c|c|c|c|c|c|c|}
\hline \multirow[b]{2}{*}{ Variable } & \multicolumn{4}{|c|}{ Degree of arachnoid descent } & \multirow{2}{*}{$\begin{array}{c}\text { r-value } \\
\text { (correlation } \\
\text { coefficient) }\end{array}$} & \multirow{2}{*}{$\begin{array}{c}\text { Sig. } \\
p \text {-value }\end{array}$} \\
\hline & No descent & $\begin{array}{l}\text { Minimal } \\
\text { descent }\end{array}$ & $\begin{array}{c}\text { Moderate } \\
\text { descent }\end{array}$ & $\begin{array}{l}\text { Marked } \\
\text { descent }\end{array}$ & & \\
\hline Overall & $13(31.7)$ & $9(22.0)$ & $7(17.0)$ & $11(29.3)$ & & \\
\hline Cavernous sinus involvement & & & & & 0.164 & 0.326 \\
\hline Knosp grade 0-2 & $11(26.8)$ & $7(17.1)$ & $6(14.6)$ & $7(17.1)$ & & \\
\hline Knosp grade $3 \mathrm{~A}$ & $0(0.0)$ & $2(4.9)$ & $1(2.4)$ & $4(9.8)$ & & \\
\hline Knosp grade $3 B$ & $0(0.0)$ & $0(0.0)$ & $1(2.4)$ & $0(0.0)$ & & \\
\hline Knosp grade 4 & $2(4.9)$ & $0(0.0)$ & $0(0.0)$ & $0(0.0)$ & & \\
\hline Tumor composition on T2WI & & & & & 0.571 & $<0.01$ \\
\hline Group A & $9(22.0)$ & $5(12.1)$ & $3(7.3)$ & $1(2.4)$ & & \\
\hline Group B & $2(4.9)$ & $2(4.9)$ & $4(9.8)$ & $1(2.4)$ & & \\
\hline Group C & $2(4.9)$ & $2(4.9)$ & $1(2.4)$ & $9(22.0)$ & & \\
\hline Displaced pituitary stalk and gland & & & & & 0.0008 & 0.962 \\
\hline Type A & $6(14.6)$ & $1(2.4)$ & $1(2.4)$ & $1(2.4)$ & & \\
\hline Type B & $1(2.4)$ & $5(12.1)$ & $2(4.9)$ & $7(17.1)$ & & \\
\hline Type C & $6(14.6)$ & $4(9.8)$ & $3(7.3)$ & $4(9.8)$ & & \\
\hline Intra-operative tumor consistency & & & & & -0.299 & 0.073 \\
\hline Soft & $8(19.5)$ & $6(14.6)$ & $5(12.1)$ & $11(26.8)$ & & \\
\hline Soft with mixed & $0(0.0)$ & $0(0.0)$ & $2(4.9)$ & $1(2.4)$ & & \\
\hline Fibrous with mixed & $3(7.3)$ & $2(4.9)$ & $1(2.4)$ & $0(0.0)$ & & \\
\hline Fibrous & $1(2.4)$ & $1(2.4)$ & $0(0.0)$ & $0(0.0)$ & & \\
\hline
\end{tabular}

Values are presented as number (\%). Sig. : significance, T2WI : T2-weighted images

Table 2. Continuous variables and correlations with degree of arachnoid descent

\begin{tabular}{lccc}
\hline Measurement & Value & r-value (correlation coefficient) & Sig. $\boldsymbol{p}$-value \\
\hline Tumor height $(\mathrm{mm})$ & $22.7 \pm 7.7(11.0-50.3)$ & 0.091 & 0.588 \\
Height of suprasellar extension $(\mathrm{mm})$ & $8.6 \pm 4.8(1.5-18.7)$ & 0.285 & 0.083 \\
Area of medial opening of DS $\left(\mathrm{mm}^{2}\right)$ & $147.9 \pm 104.9(6.8-397.5)$ & 0.340 & 0.037 \\
Area of DS $\left(\mathrm{mm}^{2}\right)$ & $336.7 \pm 121.7(181.8-666.3)$ & 0.348 & 0.032 \\
Ratio of medial opening to DS areas (\%) & $40.4 \pm 18.0(3.2-81.6)$ & 0.241 & 0.145 \\
\hline
\end{tabular}

Values are presented as mean \pm standard deviation (range) or number. Sig. : significance, DS : diaphragma sellae 
with minimal descent, seven patients (17.0\%) with moderate descent, and 11 patients (29.3\%) with marked descent.

The mean tumor height on preoperative MR imaging was $22.7 \mathrm{~mm}$ (range, 11.0 to $50.3 \mathrm{~mm}$ ). Mean tumor height, ac- cording to the degree of arachnoid membrane descent is depicted as a boxplot in Fig. 2A. These two variables were not significantly correlated $(\mathrm{r}=0.091, p=0.588)$. The mean height of suprasellar extension on preoperative MR imaging was 8.6
(A)

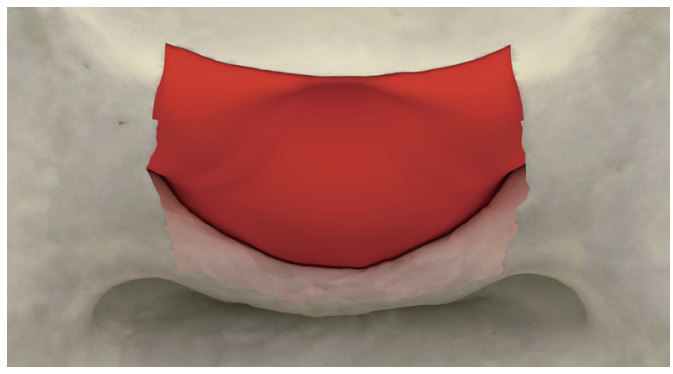

(B)

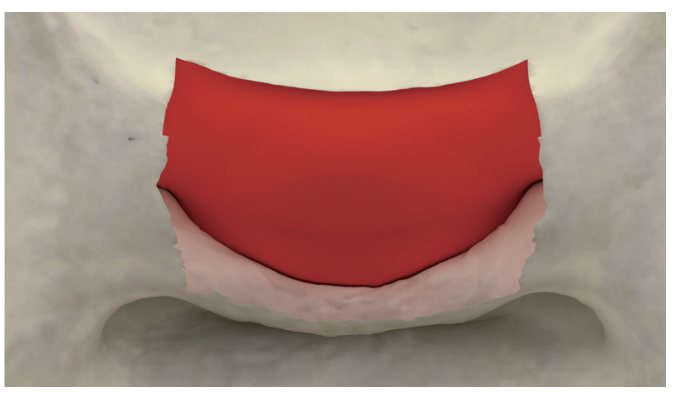

(C)

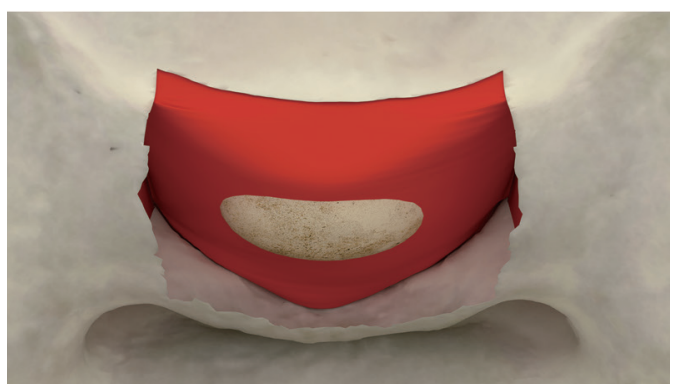

(D)

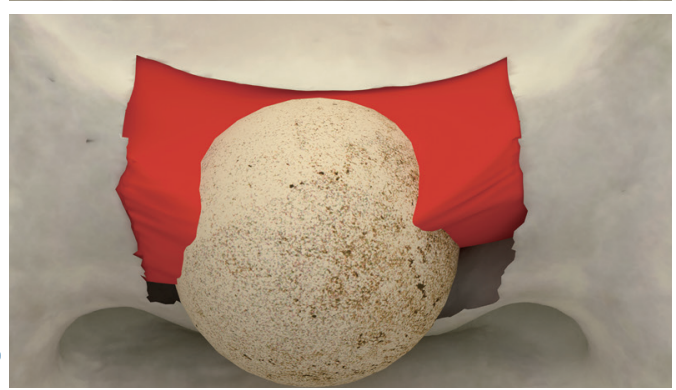

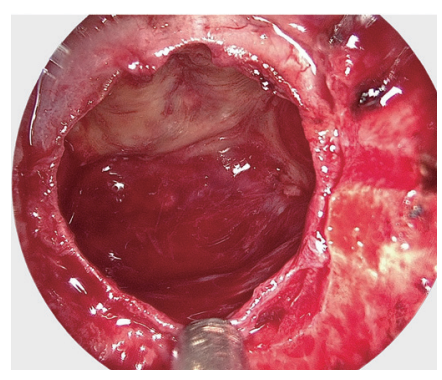
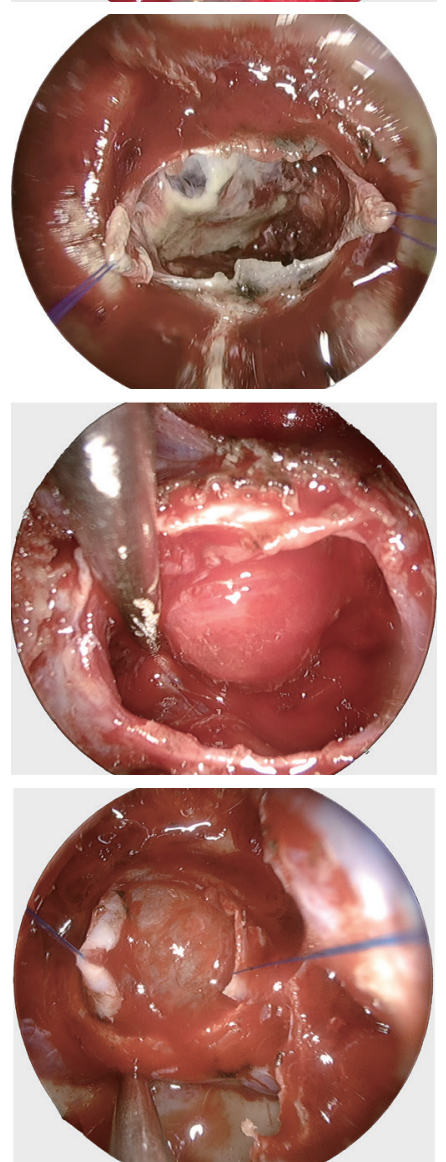
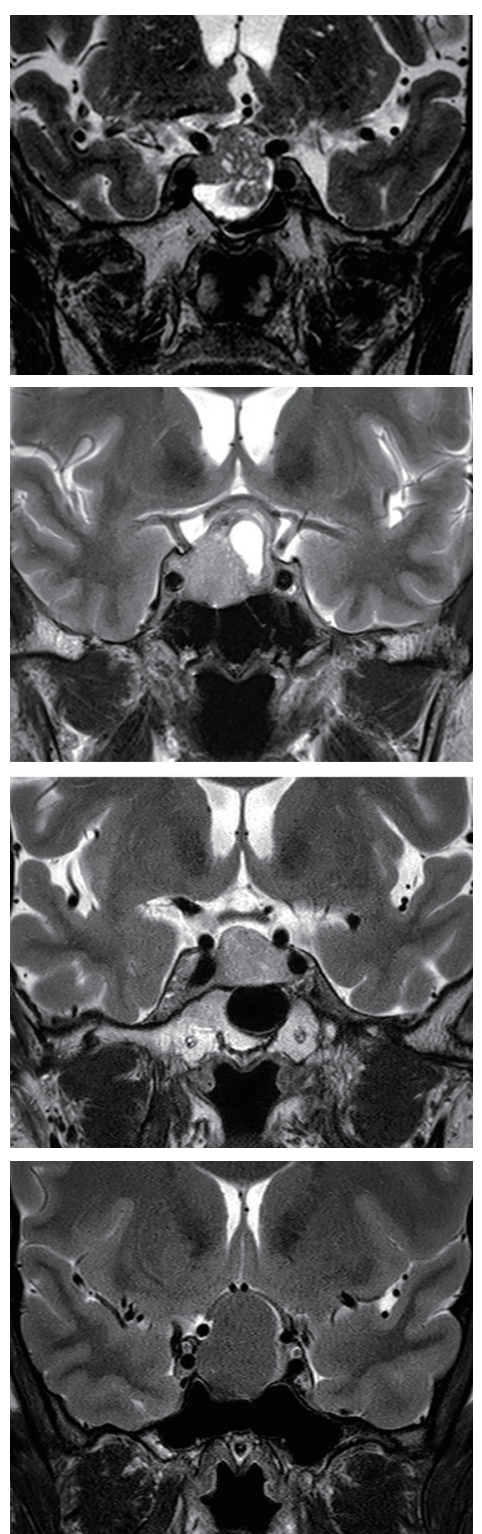

Fig. 3. Illustrations depicting the degree of arachnoid membrane descent after removal of the tumor with representative cases and corresponding T2WI. A : No descent, although oscillation of the roof structure was verified, it remained at its location without descent. The endoscopic view shows that a whitish membrane-like material covers the DS and is likely to prevent the descent of the upper structure. T2Wl of the tumor in this patient exhibits prominent mixed signal intensity. B : Minimal descent, the roof structure descended minimally. There is also a sheet-like whitish patch beneath the upper structure with a vacant space filled by the tumor on the intra-operative photograph. T2WI in this patient showed a cystic component with some mixed signal intensity. C : Moderate, the structure descended as much as the height of the suprasellar extension through the medial opening and its corresponding intra-operative endoscopic view. T2WI shows little mixed signal intensity. D : Marked descent, the structure descended more than the height of extension, and the descended arachnoid membrane is swollen and appears transparent. The intra-operative view shows the arachnoid membrane descended under the pituitary fossa, and homogenous signal intensity is exhibited on T2WI. DS : diaphragma sellae, T2WI : T2-weighted images. 
$\mathrm{mm}$ (range, 1.5 to $18.7 \mathrm{~mm}$ ). There was a trend for a correlation between these variables, but this did not reach statistical significance (Fig. $2 \mathrm{~B} ; \mathrm{r}=0.285, p=0.083$ ).

Ten patients $(24.4 \%)$ presented with true cavernous sinus infiltrations (seven patients with Knosp grade IIIa, one with IIIb, and two with IV). This parameter was not significantly correlated with arachnoid membrane descent $(r=0.164$, $p=0.326$ ). In terms of tumor composition on pre-operative T2WI, group A comprised 18 patients, group B comprised nine patients, and groups $\mathrm{C}$ comprised 14 patients. This finding is presented in a scatter plot (Fig. 2C). Tumor composition was positively correlated with the degree of arachnoid membrane descent, and it reached statistical significance $(r=0.571$, $p<0.01)$.

With regards to pituitary stalk and gland displacement, nine patients presented with type A, 15 patients with type B, and 17 patients with type $C$. These variables were not significantly correlated with arachnoid membrane descent (Fig. 2D; $\mathrm{r}=0.0008, p=0.962$ ). The area of the medial opening of the DS ranged from 6.75 to $397.49 \mathrm{~mm}^{2}$ (average, $147.9 \mathrm{~mm}^{2}$ ). DS area was 181.80 to $666.26 \mathrm{~mm}^{2}$ (average, $336.74 \mathrm{~mm}^{2}$ ). Boxplots of the areas of the medial opening of the DS and DS itself are shown in Fig. 2E and F, respectively. The ratio of the area of the medial opening to that of the DS ranged from $3.2 \%$ to $81.61 \%$ (average, $40.35 \%$ ). Both the areas of the medial opening of the DS and DS were significantly correlated with arachnoid membrane descent (area of the medial opening, $r=0.340$, $p=0.037$; area of DS, $r=0.348, p=0.032)$, although the ratio was $\operatorname{not}(\mathrm{r}=0.241, p=0.145)$ (Fig. $2 \mathrm{G}$ ).

The distribution of intra-operative tumor composition was as follows : soft (30 patients), soft with mixed contents (three patients), fibrous with mixed contents (six patients), and fibrous (two patients). Although tumor composition was not significantly correlated with arachnoid membrane descent ( $r=-0.299, p=0.073$ ), the negative gradient of the $r$ coefficient suggested that more fibrotic content was correlated with less arachnoid membrane descent after tumor removal (Fig. 2H). Correlation results for each variable are summarized in Tables 1 and 2 .

The degrees of intra-operative arachnoid membrane descent are shown with typical examples of intra-operative findings and pre-operative T2WI MR imaging depicted in Fig. 3.

\section{DISCUSSION}

The roof of the sella turcica is formed by the dual-layered dura mater, known as $\mathrm{DS}^{2,7)}$. From anterior to posterior, the DS arises from the dura covering the planum sphenoidale and extends to the dura covering the dorsum sellae. Laterally, it continues with the superior and lateral wall of the cavernous sinus. In the center, there is an opening that allows the pituitary stalk to pass from the hypothalamus to the posterior pituitary gland. This opening has been attributed varying terminology, including medial opening ${ }^{2)}$, central opening ${ }^{7,8)}$, foramen of $\mathrm{DS}^{17)}$, and diaphragm opening ${ }^{14,15)}$. Previous anatomical studies of the medial opening reported that the average maximal diameter of the medial opening was between 6.6 and $8.2 \mathrm{~mm}^{2,8,17)}$, and most are located in the posterior middle portion of the $\mathrm{DS}^{2,8)}$. As a pituitary adenoma grows to fill the sella turcica, the likely space into which the adenoma further expands would be the chiasmatic cistern through the medial opening of the DS or cavernous sinus via the medial wall of the cavernous sinus because there are sturdy barriers composed of bony structures on the anterior, inferior, and posterior surfaces of the sella turcica. In this study, we observed that a pathologic condition (pituitary macroadenoma) in this area resulted in a significant increase in the area of the medial opening. The average area of the medial opening was 147.9 $\mathrm{mm}^{2}$ (range, 6.8 to $397.5 \mathrm{~mm}^{2}$ ); this was substantially larger than the normal opening, which was estimated to be between 34.2 and $82.8 \mathrm{~mm}^{2}$ (calculated from the reported range of average maximal diameter between 6.6 and $8.2 \mathrm{~mm}$ and the assumption that the morphology was oval). The mean area of the DS, which was previously reported to be between 130.4 to $184.9 \mathrm{~mm}^{2}$ in the literature ${ }^{2,9,17)}$, also clearly increased and was $336.7 \mathrm{~mm}^{2}$ on average in this study. These increases were significantly correlated with arachnoid membrane descent through the medial opening after removal of the pituitary tumor. This correlation is not unexpected given that the relationship between resistance and cross-sectional area is inversely proportional. Nevertheless, the ratio of the area of the medial opening to that of the DS was not significantly correlated with arachnoid membrane descent. This may be the reason for which the tumor increased both the area of the medial opening and area of the DS, translating into an incremental increase in the ratio of the two areas.

The pre-operative T2WI findings of tumor composition 
were correlated with arachnoid membrane descent after tumor removal. As the tumor comprises a more homogenous material, the arachnoid membrane shows a higher tendency to descend toward the sellar floor after tumor removal. This could be because mixed signal intensities on T2WI imply that the tumors are composed of multiple constituents. As such, reactions between the inner contents may occur, resulting in the production of fibrotic tissue that finally forms a pseudocapsule. Several reports have described the presence and histology of the pseudocapsule. The pseudocapsule is located between the normal pituitary gland and adenoma ${ }^{3,4,11-13,16)}$ and comprises a wide range of products including the condensation of the basement membrane to fibroblasts, collagen fibers, and other myxoid materials ${ }^{5,6,10)}$. The etiology of pseudocapsule development has yet to be elucidated, and discussion of this process is beyond the scope of this study. Nevertheless, insight into its structural components suggests that the production of fibrotic materials could be associated with inflammatory reactions between internal constituents of the tumor, and its presence likely prevents arachnoid membrane descent after tumor removal.

Consistent with the correlation between preoperative tumor composition on T2WI and arachnoid descent, the intraoperative findings of tumor consistency also demonstrated a trend for an association with arachnoid membrane descent, although this failed to reach statistical significance. The negative r-value (correlation coefficient, -0.299) implied that softer tumor consistency led to greater descent of the arachnoid membrane. The difference between preoperative findings on T2WI and intraoperative tumor consistency may be because homogeneity on T2WI does not necessarily imply soft consistency in the operative field. Instances of mixed-signal intensity on T2WI with a dense pseudocapsule that had a soft consistency during surgery have been observed.

Guinto Balanzar et al. ${ }^{7)}$ reported four different types of DS descent during and after tumor removal in a study of 100 patients who underwent TSA for pituitary macroadenoma resection. They specifically analyzed the type of descent based on the location of the pituitary stalk and gland, and observed that tumor volume was correlated with DS descent. Therefore, we expected the deviation pattern of the pituitary stalk and gland to be correlated with arachnoid membrane descent in our study; however, this finding was not in line with our results. One possible reason for this is that their research was based on the intra-operative microscopic view and did not specify the structures that descended in the surgical bed at the end of surgery. In our study, we were able to identify the descent of the arachnoid membrane, which prolapsed through the medial opening when substantial descent of the arachnoid membrane was observed. In Guinto Balanzar et al.'s report ${ }^{7)}$, descended structures after tumor removal were presented as DS descent, which could include the arachnoid membrane, pseudocapsule of the tumor, and normal pituitary structure.

Other parameters, including tumor height, suprasellar extension, and cavernous sinus involvement, were not correlated with arachnoid membrane descent in this study. However, several reports have indicated that the descent of the DS (this term was used in previous literature) was associated with total tumor volume $e^{7)}$ or supradiaphragmatic volume ${ }^{1)}$. As this study was based on the practical estimation of size using routine imaging, we did not measure volume, as this would have necessitated specific tools or software programs. Nevertheless, we believe that the area of the medial opening and that of the DS itself may provide specific structural information, and these parameters are assumed to be proportional to tumor volume.

In this study, we used the term 'the arachnoid membrane' in describing the descending structure. At present, there is a paucity of reports that have distinctively delineated the arachnoid membrane descent associated with pituitary tumor surgery. Even among highly skilled TSA neurosurgeons, structures that have descended after tumor removal have been recognized as DS. Although DS can descend at the end of the surgery, a swollen or inflation phenomenon such as a balloon cannot be explained as DS is composed of dura mater. Moreover, a disruption in the DS would not directly lead to CSF leakage because CSF is stored within the arachnoid membrane. Therefore, we believe that the precise anatomical structure, which descends further to the sellar floor, is the arachnoid membrane.

The prediction of arachnoid membrane descent before surgery is valuable to achieve a successful TSA and prevent CSF leakage. Estimation of arachnoid membrane descent may guide surgeons in deciding whether a more extensive exploration is necessary following removal of the main bulk of the tumor. Misjudgements may result in residual tumor tissue or a torn arachnoid membrane, resulting in CSF leakage. Predicting whether the arachnoid membrane will descend readily 
will enable more attention to be paid to the handling of the thin arachnoid membrane during tumor manipulation. As the arachnoid membrane can be torn even with low powered suction, the exposed arachnoid membrane should be guarded with a cotton pad. Furthermore, if the arachnoid membrane is expected to descend easily and reach the sellar floor, it will be crucial to leave the anterosuperior portion of the tumor undisturbed until substantial removal of the posterosuperior portion of the tumor can be confirmed.

Although this preliminary analysis provides insight into methods for predicting arachnoid membrane descent before surgery, several limitations must be acknowledged. We were unable to provide precise cutoff values corresponding to arachnoid membrane descent based on our data. This was due to a technical difficulty in processing the statistics given multiple categorizations of the degree of arachnoid membrane descent. This limitation is challenging to resolve given the diverse patterns of descent of the arachnoid membrane. Furthermore, the sample size was relatively small compared to that of more extensive population-based reports. However, we attempted to minimize confounding variables by using strict exclusion criteria such as an arachnoid membrane tear during surgery or remaining tumor after surgery. We attempted to analyze intraoperative findings in every single operation via a thorough review of intraoperative video clips. Thus, we believe that this report provides useful insight into the relationship between preoperative findings and actual descent of the arachnoid membrane. Lastly, this study was designed and performed retrospectively, and data analysis from the medical records carries the risk of selection bias and errors in categorization. A well planned prospective study with a larger sample size will enable more detailed predictions of arachnoid membrane descent based on preoperative imaging.

\section{CONCLUSION}

Although extracapsular dissection is the primary strategy for pituitary adenoma removal, substantial descent of the arachnoid membrane was observed only in $46.3 \%$ (moderate descent, 17.0\%; marked descent, 29.3\%) of patients. The arachnoid membrane tended to descend more readily after tumor removal in those who had a homogeneous tumor composition on preoperative T2WI and an enlarged medial open- ing and DS area. If surgeons preparing to perform TSA for pituitary macroadenomas are able to predict the degree of arachnoid membrane descent preoperatively, complete tumor resection while avoiding tearing of the arachnoid membrane may be more feasible. Further, a well planned prospective study with a larger sample size and more structured categorization would indicate a more precise prediction of descent of the arachnoid membrane.

\section{CONFLICTS OF INTEREST}

No potential conflict of interest relevant to this article was reported.

\section{INFORMED CONSENT}

This type of study does not require informed consent.

\section{AUTHOR CONTRIBUTIONS}

\author{
Conceptualization : SHL \\ Data curation : SHL \\ Formal analysis : SHL \\ Funding acquisition : SHL, HSS \\ Methodology : SHL \\ Project administration : SHL \\ Visualization : SHL \\ Writing - original draft : SHL \\ Writing - review \& editing : HCK, SHL, HSS, JSK
}

\section{ORCID}
Hak Cheol Ko
https://orcid.org/0000-0001-8745-3309
Seung Hwan Lee
https://orcid.org/0000-0001-8043-632X
Hee Sup Shin
https://orcid.org/0000-0002-5286-8448
Jun Seok Koh 


\section{References}

1. Abdelmaksoud A, Fu P, Alwalid O, Elazab A, Zalloom A, Xiang W, et al. : Degrees of diaphragma sellae descent during transsphenoidal pituitary adenoma resection: predictive factors and effect on outcome. Curr Med Sci 38 : 888-893, 2018

2. Campero A, Martins C, Yasuda A, Rhoton AL Jr : Microsurgical anatomy of the diaphragma sellae and its role in directing the pattern of growth of pituitary adenomas. Neurosurgery 62 : 717-723; discussion 717723,2008

3. Chi JG, Lee MH : Anatomical observations of the development of the pituitary capsule. J Neurosurg 52 : 667-670, 1980

4. Ciric I: On the origin and nature of the pituitary gland capsule. J Neurosurg 46 : 596-600, 1977

5. Costello RT : Subclinical adenoma of the pituitary gland. Am J Pathol 12 : 205-216.1, 1936

6. Farnoud MR, Kujas M, Derome P, Racadot J, Peillon F, Li JY : Interactions between normal and tumoral tissues at the boundary of human anterior pituitary adenomas. An immunohistochemical study. Virchows Arch 424 : 75-82, 1994

7. Guinto Balanzar G, Abdo M, Mercado M, Guinto P, Nishimura E, Arechiga N : Diaphragma sellae: a surgical reference for transsphenoidal resection of pituitary macroadenomas. World Neurosurg 75 : 286293, 2011

8. Ju KS, Bae HG, Park HK, Chang JC, Choi SK, Sim KB : Morphometric study of the korean adult pituitary glands and the diaphragma sellae. J Korean Neurosurg Soc 47 : 42-47, 2010

9. Kursat E, Yilmazlar S, Aker S, Aksoy K, Oygucu H : Comparison of lateral and superior walls of the pituitary fossa with clinical emphasis on pitu- itary adenoma extension: cadaveric-anatomic study. Neurosurg Rev 31 : 91-98; discussion 98-99, 2008

10. Lee EJ, Ahn JY, Noh T, Kim SH, Kim TS, Kim SH : Tumor tissue identification in the pseudocapsule of pituitary adenoma: should the pseudocapsule be removed for total resection of pituitary adenoma? Neurosurgery 64(3 Suppl) : ons62-ons69; discussion ons69-ons70, 2009

11. Oldfield EH, Vortmeyer AO : Development of a histological pseudocapsule and its use as a surgical capsule in the excision of pituitary tumors. J Neurosurg 104 : 7-19, 2006

12. Peker S, Kurtkaya-Yapicier O, Kiliç T, Pamir MN : Microsurgical anatomy of the lateral walls of the pituitary fossa. Acta Neurochir (Wien) 147 : 641-648; discussion 649, 2005

13. $Q u$ X, Xu G, Qu Y, Song T: The pseudocapsule surrounding a pituitary adenoma and its clinical significance. J Neurooncol 101 : 171-178, 2011

14. Tsutsumi S, Ono H, Yasumoto $Y$, Ishii $H$ : The diaphragma sellae, diaphragm opening, and subdiaphragmatic cistern: an anatomical study using magnetic resonance imaging. Surg Radiol Anat 41 : 529-534, 2019

15. Wei L, Xi Z, Lin S, Zhao Q, Jing J, Wang $S$ : MRI research of diaphragma sellae in patients with pituitary adenoma. Int J Clin Exp Med 8 : 12842-12849, 2015

16. Yasuda A, Campero A, Martins C, Rhoton AL Jr, Ribas GC : The medial wall of the cavernous sinus: microsurgical anatomy. Neurosurgery 55 : 179-190, 2004

17. Yohannan DG, Krishnapillai R, Suresh R, Ramnarayan S : A morphometric study of the foramen of diaphragma sellae and delineation of its relation to optic neural pathways through computer aided superimposition. Anat Res Int 2015 : 618042, 2015 